which contains a summary of the available experimental information on these parameters for different rock types, the book demands a sound grasp of mathematical physics on the part of the reader. 'The chapters vary considerably in length. Chapter three by $\mathrm{R}$. R. Rumer ("Resistance to Flow through l'orous Media") and chapter five by E. S. Simpson ("Volocity and the Longitudinal Dispersion Cocfficient in Flow through Porous Media") form short yet comprohensive treatments of their subjects. Chaptor seven by D. Dicker, chapter nine by G. de Josselin de Jong and chapter ten by the editor aro longer, and require a good mathematical background to be appreciated fully.

In dealing with unsteady flows in a compressible porous medium in chapter eight, A. Verruijt roviews material drawn from the literature of both soil mechanies and hydrology. A similar interdisciplinary approach is adopted by $\mathbf{H}$. J. Morel-Seytoux in chapter eleven which draws upon experience in the petroleum industry.

Chapter six by D. Swartzendruber ("Tho Flow of Water in Unsaturated Soils") presents an interesting blend of theory and experimontal results; in this respect, this is the most balanced contribution in the book. Finally, in introducing the longest chapter, J. Bear (chapter four) takes care to point out that his review of hydrodynamic dispersion is incomplete, a testimony to the literature explosion on this aspect of porous media flow.

In general, this type of book is prone to lack of uniformity in both notation and the level of competence required of the reader. As the editor, Dr De Wiest is to be congratulated on avoiding these pitfalls. The level of presentation, however, is very high-postdoctoral rather than postgraduate. The standard is certainly above that of most one-year MSc courses, irrespective of basic discipline, and the appeal of the book secms limited to research workers in this particular field. The prospect of a small circulation is reflected in the price. M. J. HALI.

\section{COLLECTED EARTHWORKS}

The Seismicity of the Earth

1953-1965. By J. P. Rothé. (Earth Sciences, No. 1.) Pp. 336. (Unesco: Paris, 1969.) 48 francs; $\$ 12 ; 72 s$.

THIs is, against all the odds, a splendid book and one which seismologists may well feel they need themselves as well as having access to in a library. I must admit that at first glance I feared this book would be of little value. It is unashamedly a successor to Gutenberg and Richter's classic Seismicity of the Earth, and re-runs do not normally arouse much enthusiasm. Further, the recent compilation of US Coast and Geodetic Survey epicentres since 1961 by Barazangi and Dorman might seem to have saturated the subject. Not at all.

The study of scismicity is more than just a process of geographical cataloguing. Variation in activity with time, loss of life and damage to property, sorting of events by magnitude and the merging of seismic data into tectonic concepts - these are all important aspects of seismicity studies, and Professor Rothé has achieved a good balance of hard facts and general speculation. He has drawn on a wide variety of sources and his bibliography is superbnobody studying local seismicity should venture on an area until he has followed up all Rothé's leads. Further, he makes wide use of the seismological notes of the Bulletin of the Seismological Society of America-an invaluable compilation which earns too little credit for its editor.

After an intelligent introduction surveying global seismic activity, the regions of the world are reviewed in turn, maintaining the same layout as Gutenberg and Richter. Bilingual (English and French) comments follow the listing of all earthquakes betwcen 1953 and 1965 with a magnitude greater than about $6 \cdot 0$. The magnitude criterion is rolaxed, however, when small earthquakes have oecurred in unusual locations or havo wrought considerable damage. At the end of the book a set of maps show loeations, together with some notable faults and nuclear tosts.

The whole is well produced and handsomely printed. The translation is first-rate. It is difficult to find fault with this invaluable work of reference, which is going to save hours of thumbing through journals, maps and cards.

D. DAVIES

\section{TRIANGULAR PUZZLES}

\section{Geometric Inequalities}

By O. Botitema, R. Ž. Djordjević, R. R. Janić, D. S. Mitrinović and P. M. Vasić. Pp. 151. (Wolters-Noordhoff: Groningen, 1969.) $\$ 4.90$.

The book consists of a list of some four hundred inequalities on triangles and a further sixty on quadrilaterals, polygons and circles. A good many have a proof or somo indication of a proof and nearly all are elementary in nature. Some, however, would provide testing oxercises for any mathematician, even though the majority would be of most interest to secondary school students. Hardly any of the examples requiro any previous knowledge. Many are of the type which says that there is a maximum or minimum in the most symmetric case. For example, if $A, B, C$ aro the angles of a triangle, then

$$
\sin A+\sin B+\sin C \leq 3 \sqrt{3} / 2 ;
$$

or, if $h_{a}, h_{\dot{b}}, h_{c}$ are the altitudes of a triangle, then

$$
(3 r)^{3} \leqslant h_{a} h_{b} h_{c} \leqslant(3 R / 2)^{3}
$$

where $r$ and $R$ are the radii of the incircle and circumeircle respectivoly; or, the area of an $n$-gon inscribed in a unit circle is at most that of theregular one, namely, $(n / 2) \sin (2 \pi / n)$. It is mentioned that the last example was set in a high school students' competition in China in 1957. The book would be a useful addition to any school library although, it must be said, it does not greatly extend the imagination, which a book of puzzles ought to do. More precisely, even though most of the book is devotod to triangles, the more interesting examples are in the last three scctions of the book. For example, if a regular hexagon of area $F$ contains non-intersecting eircles of radii $r_{1}, \ldots, r_{n}$, then

$$
\left(r_{1}+. \cdot+r_{n}\right)^{2} \leq n F / \sqrt{12}
$$

Referenees to all the results are given. Finally, the authors are to be admired for their assiduity in achieving such a complete collection.

JAMES HiRschFELd

\section{Obituaries}

\section{Dr G. S. Carter}

George Stuart Carter was born on September 15, 1893 , the son of the Rev. G. C. Carter and Hilda E. Keane. His schooling was at Marlborough College and it was probably there that he gave his first scientific paper, as evidenced by the entry "G. S. Carter . . . Ants" in the record of the Marlborough College Natural History Socicty for February 5,1912 . This youthful endeavour was the precursor of a lifetime's work in biology.

He went up to Gonville and Caius College, Cambridge, and read for Part I of the Mathematics Tripos in 1913. The First World War then called him to serve until 1919. first in the Loicestershiro Regiment and lator in the Roya] Engineers. Returning to Cambridge he read for Part II of the Natural Sciences Tripos in Zoology, gaining a First and being awarded the Frank Smart Prize and Student. 